Pacific Northwest

National Laboratory

Operated by Battelle for the

U.S. Department of Energy

\title{
Probabilities of a Grid of Rectangular Transects of Positive Width Intersecting an Elliptical Target
}

R.F. O'Brien

April 2002

Prepared for the U.S. Department of Energy under Contract DE-AC06-76RL01830 


\title{
DISCLAIMER
}

This report was prepared as an account of work sponsored by an agency of the United States Government. Neither the United States Government nor any agency thereof, nor Battelle Memorial Institute, nor any of their employees, makes any warranty, express or implied, or assumes any legal liability or responsibility for the accuracy, completeness, or usefulness of any information, apparatus, product, or process disclosed, or represents that its use would not infringe privately owned rights. Reference herein to any specific commercial product, process, or service by trade name, trademark, manufacturer, or otherwise does not necessarily constitute or imply its endorsement, recommendation, or favoring by the United States Government or any agency thereof, or Battelle Memorial Institute. The views and opinions of authors expressed herein do not necessarily state or reflect those of the United States Government or any agency thereof.

\author{
PACIFIC NORTHWEST NATIONAL LABORATORY \\ operated by \\ BATTELLE \\ for the \\ UNITED STATES DEPARTMENT OF ENERGY \\ under Contract DE-AC06-76RL01830
}

Ty 


\section{Probabilities of a Grid of Rectangular Transects of Positive Width Intersecting an Elliptical Target}

R.F. O'Brien

April 2002

Prepared for the U.S. Department of Energy under Contract DE-AC06-76RLO1830

Pacific Northwest National Laboratory

Richland, Washington 99352 


\section{Contents}

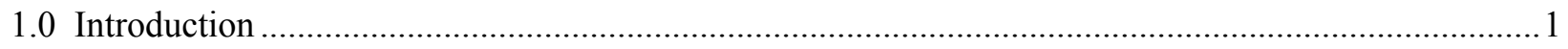

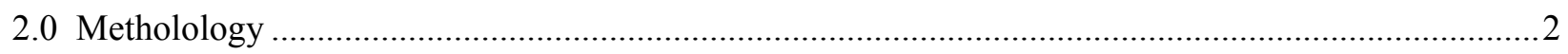

2.1 Probability of an Ellipse Intersecting an Elementary Rectangle of the Grid...........................2

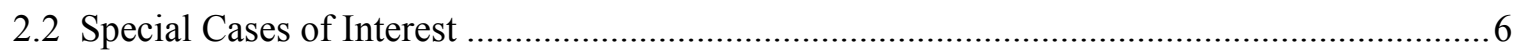

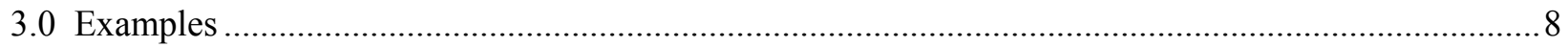

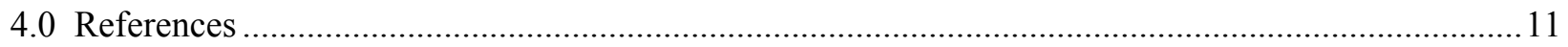




\section{Figures}

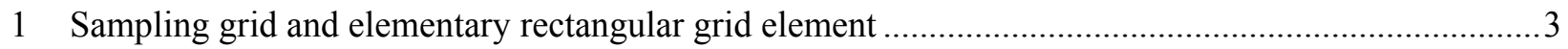

2 Elementary rectangle $T\left(a+w_{1}, b+w_{2}\right)$, rectangle $\mathrm{R}(\mathrm{a}, \mathrm{b}), \mathrm{R}\left(\mathrm{a}+\mathrm{w}_{1}, \mathrm{~b}+\mathrm{w}_{2}\right)$, and

$\mathrm{R}\left(\mathrm{a}-\mathrm{x}_{\theta}, \mathrm{b}-\mathrm{y}_{\theta}\right)$ and ellipse with semi-axes $\mathrm{r}_{1}$ and $\mathrm{r}_{2}$.....

\section{Table}

1 Probabilities of a grid with dimensions $\mathrm{a}, \mathrm{bb}$ and $\mathrm{w}$ intersecting an ellipse for specified values of $r_{1}$ and $r_{2}$ 


\subsection{Introduction}

A problem often considered in environmental characterization and remediation is to develop a sampling scheme for detecting a contaminated target area of a specified size and shape. The typical approach is to define a systematic grid pattern of sampling points that will have a specified probability of detecting a randomly located target area of concern. When the sampling points lie on the nodes of a rectangular or triangular grid of field transects and the specified target area is elliptical, Gilbert (1987) discusses a method developed by Singer $(1972,1975)$ that gives the proper grid spacing for the nodes. When the grid is rectangular and the sampling points are continuous along the transects, Duma and Stoka (1993) give a methodology to find the probability of traversing a randomly located elliptical target for the special case when both axes of the ellipse are less than the length of the smallest side of an elementary rectangle of the grid. This paper extends the results of Duma and Stoka's methodology to the more general case where the transects may also have a specified width and further includes the case where one of the axes of the ellipse may be shorter than one of the sides of an elementary rectangle of the grid.

This paper develops a methodology of finding the probability of the grid traversing an elliptical target of a specified size when a rectangular grid is used for sampling and the transects have a specified width. This probability can then be used to determine the parameters of the rectangular grid that is necessary to find a specified elliptical target with a specified probability. This methodology was developed for the situation in which a nondestructive assay device such as a magnetometer or ground penetrating radar is used to pass over the transects of a rectangular grid looking for elliptically shaped clusters of shrapnel that may indicate the existence of unexploded ordnance (UXO). In these situations, the field of view of the magnetometer or ground penetrating radar has a given width. The methodology, however, is applicable to any environmental situation where a measurement device collects continuous, or near continuous, measurements of a certain width along a transect. 


\subsection{Methodology}

To find the dimensions of the grid it is sufficient to develop a relationship that gives the probability of the grid traversing a target ellipse as a function of the parameters that define an elementary rectangle of the grid and the ellipse. This follows from the fact that if the grid traverses the target ellipse then the ellipse's center must lie in one of the elementary rectangles of the grid. Once a formula for this probability is developed, it is only a matter of solving for the unknown parameters of the elementary rectangle of the grid to find the appropriate grid spacing needed to find an ellipse of a specified size with a given probability.

Section 2.1 develops a formula for the probability of a randomly placed target ellipse intersecting an elementary rectangle of the grid in terms of the parameters that define an elementary rectangle of the grid and the ellipse. Two cases are considered: one is where the dimensions of the ellipse are such there exists a set of points inside an elementary rectangle of the grid where the ellipse can be fully rotated 360 degrees about its center without intersecting the grid; the other case is where there is no such set of points where the ellipse can be fully rotated 360 degrees about its center without intersecting the ellipse. Section 2.2 discusses certain special cases for the parameters of the grid and target ellipse. These special cases are: 1) where the grid consists of only parallel transects, 2) where the target area is a circle, 3) when the angle of orientation to the grid is known, and 4) when there may be more than one target ellipse. Section 3 gives examples using these formulas to find grid dimensions that will yield a specified probability of traversing the target ellipse when the target ellipse and transect widths are specified.

\subsection{Probability of an Ellipse Intersecting an Elementary Rectangle of the Grid}

Let $L$ be a rectangular grid in the Euclidean plane whose transects have a specific width $\mathrm{w}_{1}$ in the north-south direction and $\mathrm{w}_{2}$ in the east-west direction. Without loss of generality, let an elementary rectangle of this grid, $T\left(a+w_{1}, b+w_{2}\right)$, be defined as

$$
T\left(a+w_{1}, b+w_{2}\right)=\bigcup_{i=1}^{4} B_{i}
$$

where

$$
\begin{aligned}
& B_{1}=\{(x, y) \mid 0 \leq x<a, y=0\}, \\
& B_{2}=\{(x, y) \mid x=0,0<y<b\}, \\
& B_{3}=\left\{(x, y) \mid a \leq x<a+w_{1}, 0 \leq y<b\right\}, \\
& B_{4}=\left\{(x, y) \mid 0<x<a+w_{1}, b \leq y<b+w_{2}\right\} .
\end{aligned}
$$

Also define $R\left(a+w_{1}, b+w_{2}\right)$ as the rectangle formed by the vertices $(0,0),\left(a+w_{1}, 0\right)$, $\left(a+w_{1}, b+w_{2}\right)$ and $\left(0, b+w_{2}\right)$. Similarly define $R(a, b)$. We are interested in finding the probability of an ellipse with semi-axes $r_{1}$ and $r_{2}, r_{2} \leq r_{1}$, whose center lies inside the rectangle $R\left(a+w_{1}, b+w_{2}\right)$ that intersects an elementary rectangle $T\left(a+w_{1}, b+w_{2}\right)$ of the grid. This probability, $\mathrm{P}$, assumes that the center of the ellipse and its angle $\theta$ between its 
main axis and the side $\mathrm{a}+\mathrm{w}_{1}$ are independent uniformly distributed on $T\left(a+w_{1}, b+w_{2}\right)$ and on $[0, \pi)$ respectively. Figures 1 and 2 show a graphic of the grid and $T\left(a+w_{1}, b+w_{2}\right)$, $R\left(a+w_{1}, b+w_{2}\right), R(a, b)$ and an ellipse.

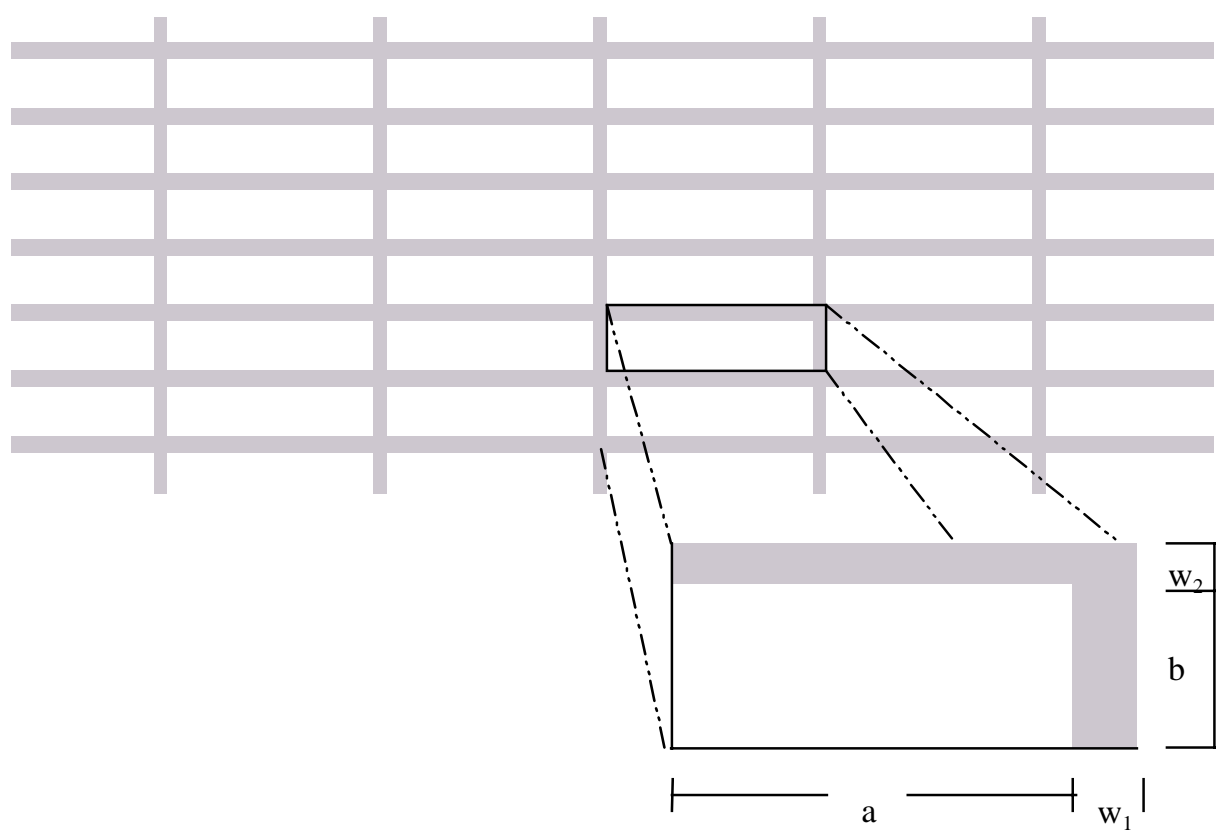

Figure 1. Sampling grid and elementary rectangular grid element. 


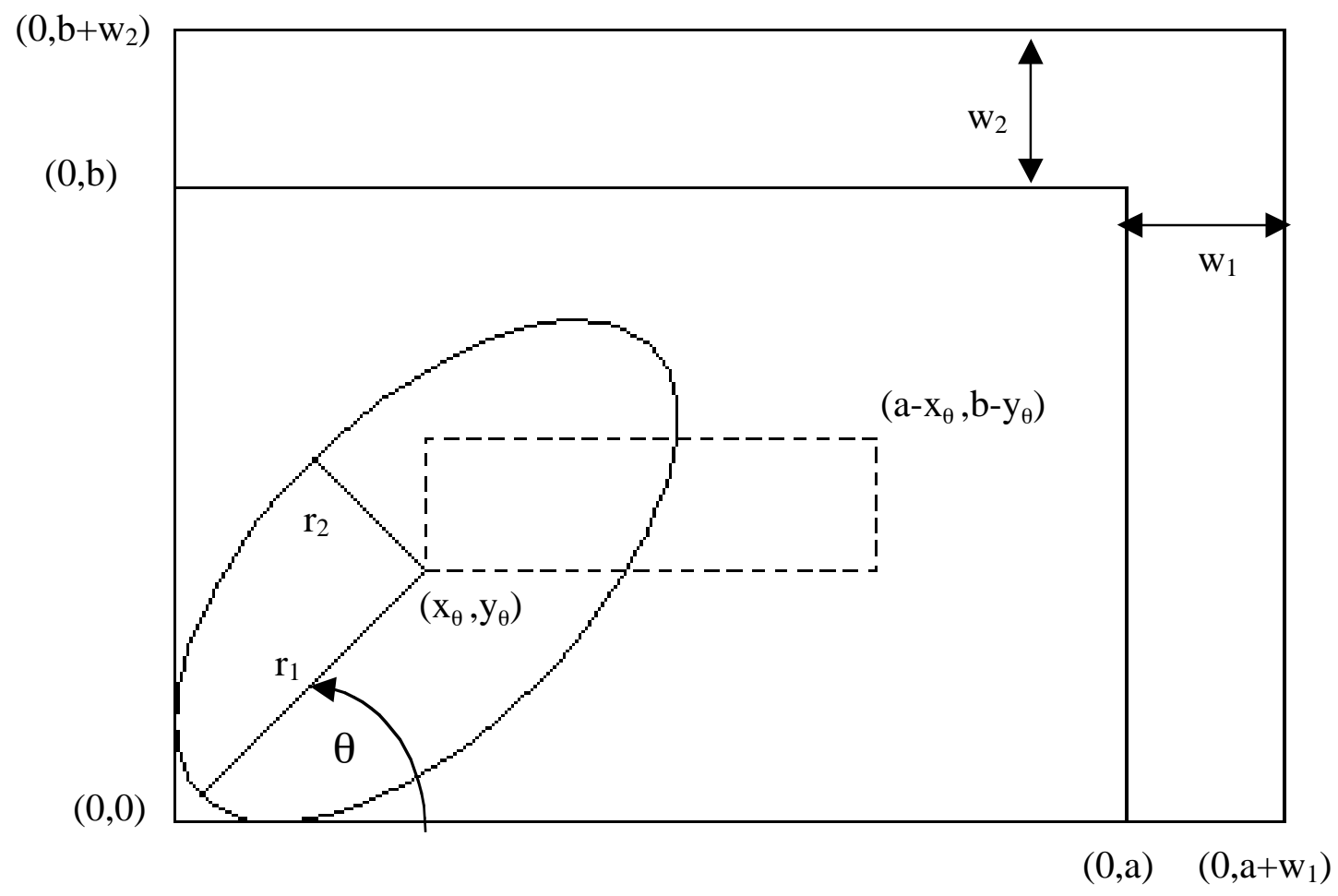

Figure 2. Elementary rectangle $T\left(a+w_{1}, b+w_{2}\right)$, rectangle $\mathrm{R}(\mathrm{a}, \mathrm{b}), \mathrm{R}\left(\mathrm{a}+\mathrm{w}_{1}, \mathrm{~b}+\mathrm{w}_{2}\right)$, and $\mathrm{R}(\mathrm{a}-$ $\left.\mathrm{x}_{\theta}, \mathrm{b}-\mathrm{y}_{\theta}\right)$ and ellipse with semi-axes $\mathrm{r}_{1}$ and $\mathrm{r}_{2}$

For a given angle $\theta$, an ellipse with its center at $\left(x_{\theta} y_{\theta}\right)$ will lie just inside the rectangle $R(a, b)$ without intersecting $R(a, b)$ when $x_{\theta}=\left(r_{1}^{2}-d^{2} \sin ^{2} \theta\right)^{1 / 2}$ and $\mathrm{y}_{\theta}=\left(r_{1}^{2}-d^{2} \cos ^{2} \theta\right)^{1 / 2}$, where $d^{2}=r_{1}^{2}-r_{2}^{2}$. This can be shown using differential calculus, noting that $-x_{\theta}$ and $x_{\theta}$ are the minimum and maximum $\mathrm{x}$ values of the ellipse with respect to $\mathrm{y}$ and $-y_{\theta}$ and $y_{\theta}$ are the minimum and maximum $y$ values of the ellipse with respect to $x$ when the ellipse has its center at the origin and whose major axis is at an angle $\theta$ to the $x$-axis. To find the probability $P$, it is sufficient to only find the probability, $Q$, that the ellipse does not intersect $R(a, b)$, since

$$
P=1-\frac{a b}{\left(a+w_{1}\right)\left(b+w_{2}\right)} Q \text {. }
$$

There are two cases to consider: one where $2 r_{2} \leq 2 r_{1}<\min \{a, b\}$ and the other when $2 r_{2}<\min \{a, b\} \leq 2 r_{1}<\max \{a, b\}$. In the first case, if the ellipse has its center in the rectangle $\mathrm{R}\left(\mathrm{a}-\mathrm{x}_{\theta}, \mathrm{b}-\mathrm{y}_{\theta},\right)$ formed by the vertices $\left(x_{\theta} y_{\theta}\right)\left(a-x_{\theta} y_{\theta}\right),\left(a-x_{\theta}, b-y_{\theta}\right)$ and $\left(x_{\theta} b-y_{\theta}\right)$ the ellipse never intersects $R(a, b)$ for all angles $\theta$, where $0<\theta<\pi$. In the second case, this is not true for all $0<\theta<\pi$.

Case 1: $2 r_{2} \leq 2 r_{1}<\min \{a, b\}$ 
To find $Q$ in this case note that an ellipse with semi-axes $r_{l}$ and $r_{2}$ and angle $\theta$ will not intersect $R(a, b)$ if the center lies in the rectangle $R\left(a-\mathrm{x}_{\theta},, \mathrm{b}-\mathrm{y}_{\theta},\right)$. In this case Duma and Stoka show that the probability of the ellipse not intersecting the rectangle $R(a, b)$ is given by

$$
\begin{aligned}
Q & =(\pi a b)^{-1} \int_{0}^{\pi}\left(a-2 x_{\theta}\right)\left(b-2 y_{\theta}\right) d \theta \\
& =(\pi a b)^{-1} \int_{0}^{\pi}\left[a-2\left(r_{1}^{2}-d^{2} \sin ^{2} \theta\right)^{1 / 2}\right]\left[b-2\left(r_{1}^{2}-d^{2} \cos ^{2} \theta\right)^{1 / 2}\right] d \theta \\
& =(\pi a b)^{-1}\left[4(a+b) r_{1} E\left(\frac{d^{2}}{r_{1}^{2}}\right)-8\left(r_{1} r_{2}\right) E\left(-\frac{d^{4}}{\left.4 r_{1}^{2} r_{2}^{2}\right)}\right)\right]
\end{aligned}
$$

where $E(\bullet)$ is a complete elliptic integral of the second kind defined as $E(z)=\int_{0}^{\pi / 2} \sqrt{1-z \sin ^{2}(\varphi)} d \varphi$. It then follows that

$$
\begin{aligned}
P & =1-\frac{a b}{\left(a+w_{1}\right)\left(b+w_{2}\right)} Q \\
& =1-\frac{\int_{0}^{\pi}\left[a-2\left(r_{1}^{2}-d^{2} \sin ^{2} \theta\right)^{1 / 2}\right]\left[b-2\left(r_{1}^{2}-d^{2} \cos ^{2} \theta\right)^{1 / 2}\right] d \theta}{\pi\left(a+w_{1}\right)\left(b+w_{2}\right)}
\end{aligned}
$$

Here numerical integration is required to obtain the desired probability. This is easiest to implement using Gaussian quadrature.

Case 2: $2 r_{2}<\min \{a, b\} \leq 2 r_{1}<\max \{a, b\}$.

In this case for a given angle $\theta$, the ellipse will not intersect $R(a, b)$ only if the center of the ellipse is in the rectangle $R\left(a-x_{\theta}, b-y_{\theta}\right)$ where $0<\theta<\cos ^{-1}\left(\sqrt{\left(\mathrm{r}_{1}^{2}-b^{2} / 4\right) / d^{2}}\right)$ or $\pi-\cos ^{-1}\left(\sqrt{\left(\mathrm{r}_{1}^{2}-b^{2} / 4\right) / d^{2}}\right)<\theta<\pi$. These values of $\theta$ are obtained by noting that the height of the rectangle $R\left(a-x_{\theta}, b-y_{\theta}\right)$, given by $b-2 y_{\theta}$, is only greater than zero when $\theta$ is in these intervals.

In this case the probability, $P$, can be obtained as

$$
\begin{aligned}
P & =1-\frac{2 \int_{0}^{\zeta}\left(a-2 x_{\theta}\right)\left(b-2 y_{\theta}\right) d \theta}{\pi\left(a+w_{1}\right)\left(b+w_{2}\right)} \\
& =1-\frac{2 \int_{0}^{\zeta}\left(a-2\left(r_{1}^{2}-d^{2} \sin ^{2} \theta\right)^{1 / 2}\right)\left(b-2\left(r_{1}^{2}-d^{2} \cos ^{2} \theta\right)^{1 / 2}\right) d \theta}{\pi\left(a+w_{1}\right)\left(b+w_{2}\right)}
\end{aligned}
$$

where $\xi=\cos ^{-1}\left(\sqrt{\left(\mathrm{r}_{1}^{2}-b^{2} / 4\right) / d^{2}}\right)$. Here again the probability requires numerical solution and Gaussian quadrature is easiest to implement. 


\subsection{Special Cases of Interest}

There are several special cases of interest which are discussed below.

1. The grid consists of parallel transects of width $\mathrm{w}$ at a distance $b$ apart

When one side of the elementary rectangle of the grid, say $a$, tends to infinity we obtain as a limiting case the probability that the ellipse intersects parallel transects of width $\mathrm{w}$ at a distance $b$ apart. The probability in this case becomes

$$
P=1-\frac{1}{\pi(b+w)}\left[2 \int_{0}^{\psi}\left(b-2\left(r_{1}^{2}-d^{2} \cos ^{2} \theta\right)^{1 / 2}\right) d \theta\right]
$$

where $\psi= \begin{cases}\pi / 2 & \text { if } 2 \mathrm{r}_{2} \leq 2 \mathrm{r}_{1}<\min \{\mathrm{a}, \mathrm{b}\} \\ \cos ^{-1}\left(\sqrt{\left(\mathrm{r}_{1}^{2}-b^{2} / 4\right) / d^{2}}\right) & \text { if } 2 \mathrm{r}_{2}<\min \{\mathrm{a}, \mathrm{b}\} \leq 2 \mathrm{r}_{1}<\max \{\mathrm{a}, \mathrm{b}\}\end{cases}$

2. The target area is a circle

In this case the semi-axes of the ellipse are equal, $r_{1}=r_{2}=r$, and the probability that the circle intersects $T\left(a+w_{1}, b+w_{2}\right)$ becomes

$$
P=\frac{2 a\left(r+w_{1}\right)+2 b\left(r+w_{2}\right)-4 r^{2}+4 w_{1} w_{2}}{\left(a+w_{1}\right)\left(b+w_{2}\right)}
$$

If in addition the side $a$ also tends to infinity, then a grid of parallel transects of width w is obtained and the probability becomes

$$
P=\frac{2(r+\mathrm{w})}{b+w}
$$

3. The angle of orientation, $\theta$, is known

If the angle of orientation of the ellipse to the grid is already known, $\theta$, then the probability of the ellipse not intersecting $R(a, b), Q$, becomes

$$
\left(\text { Area of } R\left(a-x_{\theta}, b-y_{\theta}\right)\right) /(\text { Area of } R(a, b))
$$

and the probability, $P$, becomes 


$$
\mathrm{P}=1-\frac{\left(a-2\left(\mathrm{r}_{1}^{2}-d^{2} \sin ^{2} \theta\right)^{1 / 2}\right)\left(b-2\left(\mathrm{r}_{1}^{2}-d^{2} \cos ^{2} \theta\right)^{1 / 2}\right)}{\left(a+w_{2}\right)\left(b+w_{1}\right)}
$$

where $\theta$ is an element of $(0,2 \pi)$ if $2 r_{2} \leq 2 r_{1}<\min \{a, b\}$, or $\theta$ is an element of one of the intervals $\left[0, \cos ^{-1}\left(\sqrt{\left(\mathrm{r}_{1}^{2}-b^{2} / 4\right) / d^{2}}\right)\right]$ or $\left[\pi-\cos ^{-1}\left(\sqrt{\left(\mathrm{r}_{1}^{2}-b^{2} / 4\right) / d^{2}}\right), \pi\right]$ if $2 r_{2}<$ $\min \{a, b\} \leq 2 r_{1}<\max \{a, b\}$.

4. The probability of intersecting more than one ellipse

The probability of one ellipse intersecting the grid is given above as $P$. If there is interest in there possibly being more than one ellipse of interest in the Euclidean plane then the probability of intersecting at least one of $n$ possible ellipses of the same size can be obtained as follows:

For a given ellipse and a given grid the probability of intersecting one ellipse that is randomly placed on the Euclidean plane is given by $P$ above. If there are $n$ ellipses of interest, all of the same size and independently distributed on the Euclidean plane, then the probability of not intersecting any of the ellipses with the given grid is $(1-P)^{n}$. Thus, the probability of intersecting at least one ellipse is given by $1-(1-P)^{n}$

If each of the $n$ ellipses is of a different size and independently distributed on the Euclidean plane then each ellipse has a probability of $P_{\mathrm{i}}$ of intersecting the grid where $P_{i}$ depends on the length of the semi-axes of each ellipse. Then the probability of the grid not intersecting any of the ellipses is given by $\prod_{i=1}^{n}\left(1-P_{i}\right)$ and thus the probability of the grid intersecting at least one of the ellipses is given by $1-\prod_{i=1}^{n}\left(1-P_{i}\right)$.

It should be noted that the probabilities above were mostly solved when the transect widths may be different, $\mathrm{w}_{1} \neq \mathrm{w}_{2}$, to cover the most general case. This was to cover the instances where parallel transects, say north-south, may be done at one time using some non-destructive instrument or array of instruments and the east-west transects were done at another time possibly using a different non-destructive device or array of devices. In most real applications $w_{1}=w_{2}=w$ and these values may be easily substituted into the probability calculations. 


\subsection{Examples}

To demonstrate how the probabilities above can be used to find the dimensions of a grid let us start out with a fixed size grid and a fixed size ellipse. For the target ellipse let $r_{1}=2.5$ and $r_{2}=1.0$, and let the grid parameters be $a=15.0, b=10.0$ and $w_{1}=w_{2}=0.05$. Then since $2 r_{1}<2 r_{2}<\min \{a, b\}$ we get the probability of a grid intersecting the specified ellipse to be

$$
\begin{aligned}
P & =1-\frac{1}{\pi\left(a+w_{1}\right)\left(b+w_{2}\right)}\left(\int_{0}^{\pi}\left[a-2\left(r_{1}^{2}-d^{2} \sin ^{2} \theta\right)^{1 / 2}\right]\left[b-2\left(r_{1}^{2}-d^{2} \cos ^{2} \theta\right)^{1 / 2}\right] d \theta\right) \\
& =0.53
\end{aligned}
$$

For different values of $a$ and $b$, with $w_{1}=w_{2}=0.05$ and the ellipse fixed, we can see the differing probabilities in Table 1 . Note that probabilities of greater than 0.80 are not realized until one of the lengths, $a$ or $b$, gets close to the length of the semi-major axis of $2 r_{1}$.

Table 1. Probabilities of a grid with dimensions $a, b$ and $w$ intersecting an ellipse for

\begin{tabular}{|c|c|c|c|c|c|}
\hline$a$ & $b$ & $w_{1}=w_{2}$ & $r_{1}$ & $r_{2}$ & $P$ \\
\hline 60.0 & 40.0 & 0.05 & 2.5 & 1.0 & 0.15 \\
\hline 30.0 & 20.0 & “" & “ & “" & 0.29 \\
\hline 18.0 & 12.0 & “" & “" & “" & 0.46 \\
\hline 15.0 & 10.0 & “" & “ & “" & 0.53 \\
\hline 12.0 & 8.0 & " & " & " & 0.64 \\
\hline 10.0 & 7.0 & "“ & " & "، & 0.72 \\
\hline 8.0 & 6.0 & “" & " & "، & 0.81 \\
\hline 6.0 & 5.0 & “" & "“ & “" & 0.93 \\
\hline 5.0 & 5.0 & “ & “ & “ & 0.97 \\
\hline 5.0 & 4.0 & “" & “" & “" & 0.99 \\
\hline 5.0 & 2.0 & “" & " & “" & 1.00 \\
\hline 60.0 & 40.0 & 0.05 & 1.0 & 0.5 & 0.05 \\
\hline 30.0 & 20.0 & “" & “ & “" & 0.09 \\
\hline 18.0 & 12.0 & “" & “ & “" & 0.18 \\
\hline 15.0 & 10.0 & “" & “ & “" & 0.21 \\
\hline 12.0 & 8.0 & “" & “ & “ & 0.26 \\
\hline 10.0 & 7.0 & “ & “ & “" & 0.29 \\
\hline 8.0 & 6.0 & “" & $" “$ & “" & 0.35 \\
\hline 6.0 & 5.0 & “" & " & “" & 0.43 \\
\hline 5.0 & 5.0 & “" & “" & “" & 0.47 \\
\hline 5.0 & 4.0 & “" & “" & “" & 0.52 \\
\hline 5.0 & 2.0 & “ & “ & “" & 0.77 \\
\hline 4.0 & 2.0 & “" & " & “" & 0.80 \\
\hline 3.0 & 2.0 & “" & “" & “" & 0.86 \\
\hline 3.0 & 1.5 & “" & “ & “ & 0.97 \\
\hline 2.0 & 1.5 & “" & $" “$ & “" & 0.99 \\
\hline 2.0 & 1.0 & “" & “ & “" & 1.00 \\
\hline
\end{tabular}
specified values of $r_{1}$ and $r_{2}$

a/ This probability was computed using 7-point Guassian quadrature as discussed in Abramowitz and Stegun (1972) 
In practice the transect widths $w_{1}$ and $w_{2}$ are often known in advance due to the instruments available to take measurements along the grid transects. However, even with $w$ specified there is no unique solution to find a grid spacing that intersects an ellipse of a specified size, $r_{1}$ and $r_{2}$, for a given probability $\mathrm{p}^{*}$. This is because we have one equation with two unknown parameters, $a$ and $b$, if $\mathrm{w}$ is given.

A unique solution does exist if we assume that $a$ is a multiple of $b$, that is if we can specify a constant $c$ such that $a=c b$ where $c$ is some positive real number greater than zero.

For example if the target ellipse has dimensions $r_{1}=2.5$ and $r_{2}=1.0$ as in the above example and $w_{1}=w_{2}=0.05$ and we wish the grid to intersect the ellipse with a specified probability of $p^{*}=0.95$, then if we desire to have $a=b$ (i.e. $c=1$ ) we can solve the following equation for $a$

$$
1-\frac{1}{\pi\left(a+w_{1}\right)\left(b+w_{2}\right)}\left(\int_{0}^{\pi}\left[a-2\left(r_{1}^{2}-d^{2} \sin ^{2} \theta\right)^{1 / 2}\right]\left[b-2\left(r_{1}^{2}-d^{2} \cos ^{2} \theta\right)^{1 / 2}\right] d \theta\right)=0.95
$$

This yields $a=5.23$ and $b=5.23$, which seems reasonable looking at Table 1 above. The above situation involved using the probability defined in Case 1 above. Whether or not to use the Case 1 or Case 2 probability formula can be determined by looking at the boundary conditions for $r_{1}$ and $r_{2}$ in each case, namely

Case 1: $2 r_{2} \leq 2 r_{1}<\min \{a, b\}$ versus Case 2: $2 r_{2}<\min \{a, b\} \leq 2 r_{1}<\max \{a, b\}$.

Let $a=b=\max \left\{2 r_{1}, 2 r_{2}\right\}$ which are the smallest values for $a$ and $b$ allowed in Case 1. If for these values of $a$ and $b$ if $P$ is not greater than or equal to the specified $p^{*}$ then the solution for any $a$ and $b$ will involve the Case 2 probability.

For example let $r_{1}=1$ and $r_{2}=0.5$ as in Table 1 , then $\max \left\{2 r_{1}, 2 r_{2}\right\}=2.0$. If the desired probability for the grid to intersect the ellipse is $p^{*}=0.99$ then at $a=b=2.0$ the best that Case 1 can do is to achieve a probability of $P=0.957$. Since $P<p^{*}$ it will be necessary to use the Case 2 probability formula to solve for $a$. If it is desired to have $a=4 / 3 b$ then we can solve the following equation for $b$ when $r_{1}, r_{2}, w_{1}=w_{2}=w=0.05$, and $p^{*}=0.99$

$$
0.99=1-\frac{2}{\pi\left(a+w_{2}\right)\left(b+w_{1}\right)} \int_{0}^{\xi}\left(a-2\left(r_{1}^{2}-d^{2} \sin ^{2} \theta\right)^{1 / 2}\right)\left(b-2\left(r_{1}^{2}-d^{2} \cos ^{2} \theta\right)^{1 / 2}\right) d \theta .
$$

Solving we obtain $a=2.12$ and $b=1.59$. If we desire $a=3 / 2 b$ instead, then the solution would be $a=2.21$ and $b=1.47$.

The solutions for $a$ and $b$ can be obtained by using a numerical minimization or maximization routine found in many mathematical and statistical software packages, or 
by approximating the integral using Guassian quadrature and then using numerical minimization routines or solving the resulting equations directly for $a$ or $b$. 


\subsection{References}

Abramovitz, M. and I. Stegun. 1972. Handbook of Mathematical Functions. Dover Publications, NY, NY.

Duma, A. and M. Stoka. 1993. "Hitting probabilities for random ellipses and ellipsoids." Journal of Applied Probability. 30: 971-974

Gilbert, R.O. 1987. Statistical Methods for Environmental Pollution Monitoring. John Wiley, NY.NY.

Rohatgi, V.K. 1976. An Introduction to Probability Theory and Mathematical Statistics. John Wiley, NY, NY.

Singer, D.A. 1972. ELIPGRID: A Fortran IV program for calculating the probability of success in locating elliptical targets with square, rectangle and hexagonal grids. Geocom Programs 4:1-16

Singer, D.A. 1975. "Relative efficiencies of square and triangular grids in the search for elliptically shaped resource targets." Journal of Research of the U.S. Geological Survey, 3(2):163-167 


\section{Distribution}

No. of

$\underline{\text { Copies }}$

\section{OFFSITE}

3 DoD/SERDP
A. Andrews
M. Chambers
J. Fairbanks
Gannett Fleming
L. Wrench

No. of

$\underline{\text { Copies }}$

\section{ONSITE}

D.J. Bates

D.K. Carlson

R.O. Gilbert

R.F. O'Brien (5)

B.A. Pulsipher

G.A. Sandness

J.E. Wilson

Distr.1 